\title{
TI.29.1
}

\section{Steward Program Proof of Concept Six-Month Status Report}

- PDF: Steward Program Proof of Concept Six-Month Status Report.pdf

- Text: Steward Program Proof of Concept Six-Month Status Report.txt

\section{More Information}

\begin{tabular}{|c|c|}
\hline Repository ID & Tl.29.1 \\
\hline Persistent URL & http://doi.org/10.26869/TI.29.1 \\
\hline Title & Steward Program Proof of Concept Six-Month Status Report \\
\hline Authors & Mark Johnson, John Krienke, Kevin Morooney, Mark Scheible, David Walker, Ann West, Mike Zawacki \\
\hline Sponsor & Internet2, MCNC \\
\hline \multicolumn{2}{|l|}{ Review } \\
\hline Status & Preserve \\
\hline Publish Date & 7/25/2017 \\
\hline DOI & 10.26869/TI.29.1 \\
\hline \multicolumn{2}{|l|}{ Signature } \\
\hline Deprecated & No \\
\hline \multicolumn{2}{|l|}{ Future Review } \\
\hline \multicolumn{2}{|l|}{ Supersedes } \\
\hline Format & PDF \\
\hline \multicolumn{2}{|l|}{ Related Docs } \\
\hline \multicolumn{2}{|l|}{ Development Location } \\
\hline IP Framework & CC BY 4.0 \\
\hline Subject Tags & steward, incommon, identity federation \\
\hline Notes & \\
\hline
\end{tabular}

